\title{
Current clinical application of serum biomarkers to detect ovarian cancer
}

\author{
Marek Nowak, Łukasz Janas, Grzegorz Stachowiak, Tomasz Stetkiewicz, Jacek R. Wilczyński \\ Department of Gynecology and Gynecologic Oncology, Polish Mother's Memorial Hospital - Research Institute, Lodz, Poland
}

\begin{abstract}
For the last decades, hundreds of potential serum biomarkers have been assessed in diagnosing of ovarian cancer including the wide spectrum of cytokines, growth factors, adhesion molecules, proteases, hormones, coagulation factors, acute phase reactants, and apoptosis factors but except CA125 none of them have been applied to everyday clinical practice. Nowadays, the growing number of evidence suggests that the classic marker CA125 should be accompanied by HE4 and in fact, Risk of Ovarian Malignancy Algorithm (ROMA) is becoming more and more widespread in clinical practice for the evaluation of adnexal masses. Early ovarian cancer is often asymptomatic, so the challenge still exists to develop serum markers suitable for early diagnosis and screening. Current knowledge strongly points to different mechanisms of pathogenesis, genetic disturbances and clinical course of major histological subtypes of ovarian cancer. Thus, future biomarker/multimarker panels should take into consideration the implications of different molecular patterns and biological behavior of various subtypes of ovarian cancer. Very promising are studies on miRNAs - small non-protein coding generegulatory RNA molecules functionally involved in the pathogenesis of cancers acting as oncogenes (oncomirs) or tumor suppressors. The studies devoted to ovarian cancer tissue miRNA profiling have shown that miRNAs could be useful in diagnosing and predicting the OC outcome. They also confirmed that OC is a highly heterogeneous disease, gathering four distinct histological tumor subtypes characterized not only by distinct origin, behavior and response to chemotherapy but also by different patterns of miRNA expression.
\end{abstract}

Key words: ovarian cancer, tumor markers, CA125, HE4, ROMA, OVA1, miRNAs.

\section{Introduction}

Approximately $20 \%$ of women are expected to suffer from an adnexal mass at least once in their lifetime [1]. About half of them undergo surgery for this reason [2]. Population risk of ovarian cancer is about $1-1.5 \%$, what means that the great majority of adnexal tumors is benign and can be operated by general gynecologists [3]. However, many benefits have been proven for patients with ovarian cancer operated by oncological gynecologists compared with general gynecologists and surgeons (more accurate staging, more precise cytoreductive surgery, smaller number of complications, higher percentage of 5-year survival) [4-6]. Therefore, women with suspected ovarian tumors should be directed to centers specializing in oncological gynecology.

On that ground, new methods of selecting patients at a high risk of ovarian malignancy are investigated. Nowadays, physical bimanual examination, gynecological ultrasound imaging and serum biomarkers are used to assess adnexal masses. Some authors question the utility of ultrasound because of its subjectivity and dependence on sonographer's experience [7]. Consequently, the importance of serum markers in the diagnosis of ovarian cancer is growing as they are more objective and comparable.

In the past years, a wide spectrum of cytokines, growth factors, adhesion molecules, proteases, hormones, coagulation factors, acute phase reactants, apoptosis factors were investigated as potential single serum biomarkers and in multimarker panels in diagnosing ovarian cancer, but except CA125, none of them have been applied to everyday clinical practice.

\section{CA 125}

CA125 (cancer antigen 125) is a glycoprotein, encoded by MUC16 gene on chromosome 19. Its upper limit of normal value is set at $35 \mathrm{Ul} / \mathrm{ml}$. Expression of CA125 is elevated in $85 \%$ of serous, $65 \%$ of endometrioid, $40 \%$ of clear-cell, $36 \%$ of undifferentiated and only $12 \%$ of mucinous ovarian cancers [8]. For the last three decades it has become the most widespread biomarker of ovarian cancer. The utility of CA125 in the diagnosis of ovarian cancer has been evaluated in a large number of studies.

Serum levels of CA125 are within the normal limits in at least $20 \%$ of patients with ovarian cancer and about 
a half of patients in its early stages, which considerably reduces the sensitivity of this marker. Moreover, CA125 concentrations are elevated in many non-malignant conditions, which significantly affect its specificity. The most important cause of false-positive results of CA125 is endometriosis (in about two-thirds of patients with endometriotic cysts, CA125 levels exceed the normal range) $[8,9]$. CA125 levels are also raised in patients with other gynecological diseases (such as myomas of the uterus, benign and borderline ovarian tumors), many non-gynecological illnesses (e.g. hepatic cirrhosis, congenital heart defects), during pregnancy and in $1-5 \%$ of healthy women [8, 10-12]. Because of the aforementioned limitations of CA125 protein, a large number of researches have been conducted to improve the ovarian cancer diagnostic protocol.

Attempts were made to combine CA125 with ultrasound imaging, resulting in the development of many diagnostic algorithms [13]. One of them, the Risk of Malignancy Index (RMI) has been applied in clinical practice. Four variations of RMI have been developed, first of which (RMI I) occurred to be the most efficient [14-17]. It is calculated with the use of following formula:

$$
\mathrm{RMI}=\mathrm{U} \times \mathrm{M} \times \mathrm{CA} 125,
$$

where: $U$ - ultrasound image (1 point for each of the features: solid, multilocular, bilateral tumor, ascites, intra-abdominal metastases); $U=0$ (0 points), $U=1$ (1 point), $U=3$ (2-5 points),

$M$ - menopausal status; $M=1$ (premenopausal), $M=3$ (postmenopausal),

CA125 - serum CA125 concentration $(\mathrm{U} / \mathrm{ml})$.

RMI values > 200 qualify patients to the group of high risk of malignancy, reaching the sensitivity of $78 \%$ at the specificity of $87 \%$ [13]. RMI has become a practical diagnostic tool, which is still used in the diagnosing of ovarian tumors.

\section{HE4}

HE4 (human epididymis protein 4) is a glycoprotein encoded by WFDC2 gene (chromosome 20) [18]. Presumably, it takes part in immune response, but its role has not been precisely specified yet $[19,20]$. It is present in the epithelium of fallopian tubes, endometrium, endocervical glands, but not in ovarian surface epithelium. Expression of HE4 has also been noted in epithelium of the respiratory tract (especially trachea), renal convoluted tubules and salivary glands [21, 22]. An elevated expression of HE4 is observed in $93-100 \%$ of serous, $80-100 \%$ of endometrioid and $50-83 \%$ of clear-cell carcinomas of the ovary, while it is absent in mucinous ovarian cancer [21, 22].

In preliminary studies HE4 has proved to be more sensitive (at preset specificity) than any other ovar- ian cancer marker, including CA125. It was elevated in over $50 \%$ of ovarian cancer patients with CA125 levels within normal limits. A combination of both CA125 and HE4 had higher sensitivity than any other marker combination. And HE4 achieved highest sensitivity among all tested proteins in the diagnosis of early ovarian cancer [1].

HE4 proved to be less frequently elevated than CA125 in benign ovarian tumors, both in pre- and postmenopausal women. Most of all, HE4 levels exceed normal ranges much less frequently than CA125 in cases of endometriosis (3\% vs. 67\%) [9]. Serum concentrations of HE4 are also more rarely increased than CA125 in serous cysts, teratomas, fibromas, inflammatory lesions. However, serum levels of both CA125 and HE4 do not show an statistically relevant difference in patients with mucinous cysts [9]. Because HE4 levels are not affected by many benign diseases which increase concentrations of CA125, the new marker may be a valuable complement in distinguishing malignant from benign ovarian tumors.

In contrast to CA125, HE4 concentrations are lower in pregnant women when compared with their premenopausal counterparts. Levels of HE4 do not differ significantly between trimesters. Only between second and third trimester of pregnancy, a slight statistically insignificant increase in the HE4 concentrations was reported [23].

It has been proven that hormonal therapy does not affect HE4 serum levels. Therefore, oral contraception, treatment of menstrual disorders and endometriosis do not need to be aborted for HE4 testing. The influence of hormonal replacement therapy on HE4 concentrations has not been defined well yet [24].

HE4 levels do not alter during the menstrual cycle, so they can be determined regardless of its phase [24]. Among healthy women, concentrations of HE4 increase with age (starting from the age of 40 ), especially in the $8^{\text {th }}$ and $9^{\text {th }}$ decade of life. This fact ought to be taken into account, when serum of elderly women is tested, because elevated levels of HE4 may lead to false-positive results in this group of patients [23].

Unfortunately, the normal limits for HE4 have not been established well yet. Depending upon the study, normal ranges vary from 70 to $150 \mathrm{pM}[1,25]$. Moore et al. have determined normal values separately for patients before and after menopause (89.1 pM and 128.0 pM, respectively) [23].

\section{ROMA}

Based on the encouraging results of HE4 in the diagnosis of ovarian cancer, especially in combination with CA125, Moore et al. have developed the Risk of Ovarian Malignancy Algorithm (ROMA) [7]. It utilizes serum concentrations of both CA125 and HE4, which are 
substituted to the mathematical formula, elaborated separately for pre- and postmenopausal patients.

Premenopausal patients:

$$
\mathrm{PI}=-12.0+2.38 \times \mathrm{LN}(\mathrm{HE} 4)+0.0626 \times \mathrm{LN}(\mathrm{CA} 125)
$$

Postmenopausal patients:

$\mathrm{PI}=-8.09+1.04 \times \mathrm{LN}(\mathrm{HE} 4)+0.732 \times \mathrm{LN}(\mathrm{CA} 125)$

$$
\text { ROMA }(\%)=\exp (\mathrm{PI}) /[1+\exp (\mathrm{PI})] \times 100 \%
$$

According to the cut-off values established by the authors, ROMA value $>13.1 \%$ in pre- and $>27.7 \%$ for postmenopausal women, qualified them to a group with a high risk of malignancy of ovarian tumor. Cut-off values differ slightly depending on the manufacturer of the diagnostic kit.

Authors of this algorithm revealed its sensitivity at the level of $93.8 \%$ (88.9\% for pre- and $94.6 \%$ for postmenopausal women) at the specificity of $75 \%$ in the diagnosis of epithelial ovarian cancer [7]. When compared with RMI, ROMA demonstrated higher sensitivity in diagnosis of ovarian cancer $(94.3 \%$ vs. $84.6 \%$ at $75 \%$ specificity). When early stages of ovarian cancer (FIGO I/II) were concerned, the difference between these two tests was even more remarkable $(85.3 \%$ for ROMA vs. $64.7 \%$ for RMI at $75 \%$ specificity) [26].

Many studies evaluating utility of HE4 and ROMA in the diagnosis of ovarian cancer have been published. Although most of them confirm the effectiveness of both methods, according to authors of some studies adding these methods to the diagnostic protocol is not clearly justified [27, 28]. Most of available meta-analyses show similar findings [29-31]. According to Lin et al., ROMA is more sensitive than CA125 and HE4 in the diagnosis of ovarian cancer (sensitivity of $87 \%$ at specificity of $82 \%$ ). Surprisingly, CA125 reveals even higher sensitivity than HE4 ( $80 \%$ vs. $74 \%$ ). Most importantly, HE4 proved to be the most specific of all three methods (specificity of $87 \%$ for HE 4 vs. $82 \%$ for ROMA vs. $76 \%$ for CA125) [31]. However, due to heterogeneity of studies, it is stressed that the results of meta-analyses should be treated with caution [29-31].

\section{OVA1}

In 2009, the U.S. Food and Drug Administration approved for clinical use a new test, OVA1 [32]. It evaluates serum concentrations of five markers. Two of them are upregulated (CA-125 II, $\beta$-microglobulin) and three are downregulated (apolipoprotein A1, prealbumin, transferrin) in patients with ovarian cancer. Serum levels are compiled with the use of a computer program - OvaCalc ${ }^{\oplus}$, giving a result as a number between 0 and 10 . The value $\geq 5.0$ in premenopausal and $\geq 4.4$ in postmenopausal women qualify patients to the high risk group. Patients with a positive result of OVA1 test should be referred to an oncological gynecologist [33].
Results of a multicenter study (OVA500 Study) showed sensitivity of the OVA1 test in the diagnosis of ovarian cancer at the level of $96 \%$ (91\% in FIGO I/II) and specificity of $51 \%$. Also a high negative predictive value of OVA1 test is noteworthy [34].

A little number of studies evaluating utility of the OVA1 test have been published. However, available publications confirm to some extent results of the OVA500 Study. According to one of the authors, OVA1 reveals sensitivity and specificity at the level of $96 \%$ and $28 \%$ in postmenopausal and $85 \%$ and $40 \%$ in premenopausal women, respectively [35]. In one study, OVA1 qualified to the high risk group $76 \%$ of patients with malignant adnexal tumors who had serum CA125 levels within the normal limits [36]. Some reports also confirm high sensitivity of the OVA1 test in the diagnosis of early ovarian cancer [37]. Up till now, there are hardly any available studies directly comparing performance of the OVA1 test and ROMA.

\section{Future perspectives: circulating miRNAs}

The last decade has brought better understanding of heterogeneity of ovarian cancer. Future, improved biomarker and multimarker panels should be based on molecular origin of various OC subtypes. Very promising are studies on miRNAs - small non-protein coding gene-regulatory RNA molecules functionally involved in the pathogenesis of cancers acting as oncogenes (oncomirs) or tumor suppressors.

The studies devoted to ovarian cancer tissue miRNA profiling have shown that miRNAs could be useful in diagnosing and predicting the OC outcome [38, 39]. They also confirmed that $\mathrm{OC}$ is a highly heterogeneous disease, gathering four distinct histological tumor subtypes characterized not only by distinct origin, behavior and response to chemotherapy but also by different patterns of miRNA expression [40]. From the clinical and practical point of view a more interesting and important question is whether the miRNAs could be used as attainable biomarkers (alone or combined with other markers i.e. CA125) present in peripheral blood.

MiRNA can pass between tissues and organs through blood circulation. The source of miRNAs found in the peripheral blood of cancer patients is tumor tissue. Circulatory miRNAs originate mainly from monocytes and exosomes/microvesicles released from the tumor $[41,42]$. miRNAs are incorporated into a membrane-enclosed complex or bound to proteins, therefore, are resistant to plasma RNases and stable against temperature and $\mathrm{pH}$ changes $[43,44]$. These features make them reliable candidates for diagnostic and predictive biomarkers. In most opinions circulating miRNAs are malignancy-type specific and are characterized by the same miRNA signature as the parental tumor [45]. Therefore, the majority of circulating miRNA biomarker 
studies are based on the primary tumor miRNA expression profiles. However, the lack of correlation between paired tissue-plasma miRNA expression profiles in some studies strongly suggests that malignant tumor cells are not the sole source of circulating miRNAs. It is possible that circulating miRNAs signatures are affected and changed by loco-regional inflammation observed in many tumors, and that the final result of miRNA profiling in blood is a mixture of tumor-specific and inflammation-specific miRNAs [46].

The first attempts to use serum miRNA as cancer biomarkers were described in patients with diffuse large B-cell lymphoma and indicated that levels of miR-21 correlated with patients' relapse-free survival [47]. Subsequently, serum miRNAs were tested as biomarkers for monitoring in prostate cancer (miR-141) [41], followed by studies of miRNA use for the early detection both in the lung and colorectal cancer based on miR-25 and miR-223 serum concentrations [43, 48]. Nowadays, similar studies have been performed in many types of cancer including breast cancer, gastric cancer and ovarian cancer (OC) [48, 49].

The first study in OC was performed by Taylor et al. [50] who found that miRNAs over-expressed in serous ovarian cancer tissue (miR-21, miR-141, miR-200a, miR-200c, miR-200b, miR-203, miR-205, and miR-214) were similarly elevated in serum-derived exosomes. The results differed significantly when compared to the control group of patients with benign ovarian pathology [50]. In another study, Resnick et al. [14] tested serum miRNA expression of twenty one miRNAs selected from the epithelial ovarian cancer profile. They found that five miRNAs were overexpressed (miR-21, miR-29a, miR-92, miR-93, and miR-126) and three were underexpressed (miR-127, miR-155, and miR-99b) in the pre-surgical serum of ovarian cancer patients when compared to normal controls. What is interesting, three of miRNAs, namely miR-21, miR-92 and miR-93, which probably functioned as the oncomirs, were significantly overexpressed in patients with normal pre-operative CA125 levels. This suggests that some miRNAs could be complementary biomarkers in patients with negative results of routine tests $[39,45,48]$. Overexpression of members of miR-200 family (miR-200a, miR-200b, and miR-200c) was confirmed in serum of serous OC patients by Kan et al. [51]. The predictive model constructed on the basis of miR-200 expression, in the opinion of authors, was able to discriminate patients with high-grade serous $O C$ from age-matched healthy controls [51]. According to some investigators, the use of the miR-200 family is a promising direction in diagnosis and monitoring of therapy in OC patients [45]. The research performed by Chung et al. [52] was planned to examine in the microarray analysis and qRT-PCR a total RNA isolated from serum, tissue and ascites in serous OC patients. Expression of four serum
miRNAs (miR-132, miR-26a, let-7b, and miR-145) was found to be significantly down-regulated in OC patients compared to controls, making these miRNAs potential candidates for novel biomarkers in serous OC [52]. An interesting study conducted by Suryawanshi et al. [46] compared the plasma miRNA profiles in endometriosis, endometrioid and serous ovarian cancer. The results of this study confirmed that endometrioid and serous $O C$ are distinct entities and they can be distinguished based on plasma miRNA profile. Moreover, a trend of miRNA changes from endometriosis to endometrioid OC was shown [46]. A combination of miR-21, miR-362-5p, and miR-1274a enabled differentiation between endometriosis and endometrioid OC (57\% sensitivity, 91\% specificity), while miR-21, miR-191, and miR-1975 together could distinguish between endometrioid and serous OC ( $86 \%$ sensitivity, $79 \%$ specificity) [46]. The other combinations of miRNAs could further differentiate the studied population, as follows: miR-16, miR-191 and miR-195 - healthy vs. endometriosis; miR-16, miR-21, and miR-191 - healthy vs. endometrioid OC; miR-16, miR-191, and miR-4284 - healthy vs. serous OC; miR-362-5p, miR-628-3p, and miR-1915 - endometriosis vs. serous OC [46]. Recently Ji et al. [53] identified eleven up-regulated and nineteen down-regulated miRNAs in sera of OC patients. Among them miR-22, miR-93, mir-106b and miR-451 were validated quantitatively. It was found that miR-22 and miR-93 were consistently up-regulated more than 2-fold in OC cases. Expression of miR-106b was significantly lower, whereas that of miR-451 was higher in the group of patients over 51 years of age, what was a novel and interesting discovery. Moreover, authors showed that miR-106b and miR-93 serum concentrations were down-regulated in patients with the highest CA125 levels, although there was no connection with the clinical stage of the disease [53]. Another recent study by Shapira et al. [54] for the first time focused on protein-bound miRNA in plasma free of cellular debris, microvesicles or exosomes. Analysis showed that nineteen miRNAs were downregulated while three were up-regulated in serous OC compared to control healthy subjects. Among them six miRNAs (miR-106b, miR-126, miR-150, miR-17, miR20a, and miR-92a) were significantly decreased in OC patients [54]. The results of all cited above studies are summarized in Table I (adopted from [45] and updated).

\section{Conclusions}

For the last decades hundreds of potential serum biomarkers have been assessed in diagnosing of ovarian cancer. Nowadays, the growing number of evidence suggests that the classic marker CA125 should be accompanied by HE4 and in fact, ROMA algorithm is becoming more and more widespread in clinical practice for the evaluation of adnexal masses. The second mul- 
Tab. I. Studies on circulating miRNAs as potential biomarkers of ovarian cancer

\begin{tabular}{|c|c|c|c|c|c|c|}
\hline Author/Reference no. & Year & $\begin{array}{c}\text { Ovarian } \\
\text { cancer type }\end{array}$ & Control & $\begin{array}{l}\text { Type of } \\
\text { sample }\end{array}$ & Up-regulated & Down-regulated \\
\hline $\begin{array}{l}\text { Taylor and Gercel- } \\
\text { Taylor [50] }\end{array}$ & 2008 & Serous & Benign ovarian tumors & $\begin{array}{l}\text { Exo- } \\
\text { somal } \\
\text { serum } \\
\text { miRNA }\end{array}$ & $\begin{array}{l}\text { miR-21, miR-141, } \\
\text { miR-200a, miR-200b, } \\
\text { mir-200c, miR-203, } \\
\text { miR-205, miR-214 }\end{array}$ & \\
\hline Resnick et al. [48] & 2008 & $\begin{array}{l}\text { Generally epi- } \\
\text { thelial ovar- } \\
\text { ian cancer }\end{array}$ & Healthy controls & $\begin{array}{l}\text { Serum } \\
\text { miRNA }\end{array}$ & $\begin{array}{l}\text { miR-21, miR-92, } \\
\text { miR-93, miR-126, } \\
\text { miR-29a }\end{array}$ & $\begin{array}{l}\text { miR-155, miR-127, } \\
\text { miR-99b }\end{array}$ \\
\hline Kan et al. [51] & 2012 & $\begin{array}{l}\text { Serous (high } \\
\text { grade) }\end{array}$ & Healthy controls & $\begin{array}{l}\text { Serum } \\
\text { miRNA }\end{array}$ & $\begin{array}{l}\text { miR-200a, } \\
\text { miR-200b, } \\
\text { miR-200c }\end{array}$ & \\
\hline Chung et al. [52] & 2013 & Serous & Healthy controls & $\begin{array}{l}\text { Serum } \\
\text { miRNA }\end{array}$ & & $\begin{array}{l}\text { miR-132, miR-26a, } \\
\text { Let-7b, mir-145 }\end{array}$ \\
\hline Suryawanshi et al. [46] & 2013 & $\begin{array}{l}\text { Endometri- } \\
\text { oid/Serous }\end{array}$ & $\begin{array}{l}\text { Healthy controls/Endome- } \\
\text { triosis }\end{array}$ & $\begin{array}{l}\text { Plasma } \\
\text { miRNA }\end{array}$ & $\begin{array}{l}\text { miR-16, miR-21, } \\
\text { miR-191, miR-4284 }\end{array}$ & \\
\hline Ji et al. [53] & 2014 & $\begin{array}{l}\text { Generally epi- } \\
\text { thelial ovar- } \\
\text { ian cancer }\end{array}$ & $\begin{array}{l}\text { Healthy controls/Benign } \\
\text { ovarian tumors }\end{array}$ & $\begin{array}{l}\text { Serum } \\
\text { miRNA }\end{array}$ & miR-22, miR-93 & \\
\hline Shapira et al. [54] & 2014 & Serous & $\begin{array}{l}\text { Healthy controls/Benign } \\
\text { ovarian tumors }\end{array}$ & $\begin{array}{l}\text { Plasma } \\
\text { miRNA }\end{array}$ & & $\begin{array}{l}\text { miR-106b, } \\
\text { miR-126, miR-150, } \\
\text { miR-17, miR-20a, } \\
\text { and miR-92a }\end{array}$ \\
\hline
\end{tabular}

timarker panel approved by FDA - OVA1 is not widely used outside the USA. Taken together, clinical examination, ultrasonography and serum markers (ROMA) more accurately select the patients with a high risk of ovarian malignancy what allows to direct them to centers specializing in oncological gynecology.

Such diagnostic workup is possible when the woman comes to the gynecologist and the adnexal mass is diagnosed. Ovarian cancer at the beginning gives unspecific mild signs or no symptoms at all. In fact early ovarian cancer often is asymptomatic, the challenge still exists to develop serum markers suitable for early diagnosis and screening.

Current knowledge strongly points to different mechanisms of pathogenesis, genetic disturbances and clinical course of major histological subtypes of ovarian cancer. Thus, future biomarker/multimarker panels should take into consideration the implications of different molecular patterns and biological behavior of major subtypes of OC.

\section{Disclosure}

Authors report no conflict of interest.

\section{References}

1. Moore RG, Brown AK, Miller MC, et al. The use of multiple novel tumor biomarkers for the detection of ovarian carcinoma in patients with a pelvic mass. Gynecol Oncol 2008; 108: 402-408.

2. Curtin JP. Management of the adnexal mass. Gynecol Oncol 1994; 55 (3 Pt 2): S42-46.

3. Berek JS, Novak E. Ginekologia. Tom IV. Medipage, Warszawa 2008.
4. Giede KC, Kieser K, Dodge J, Rosen B. Who should operate on patients with ovarian cancer? An evidence-based review. Gynecol Oncol 2005; 99: 447-461.

5. Earle CC, Schrag D, Neville BA, et al. Effect of surgeon specialty on processes of care and outcomes for ovarian cancer patients. J Natl Cancer Inst 2006; 98: 172-180.

6. Engelen MJ, Kos HE, Willemse PH, et al. Surgery by consultant gynecologic oncologists improves survival in patients with ovarian carcinoma. Cancer 2006; 106: 589-598.

7. Moore RG, McMeekin DS, Brown AK, et al. A novel multiple marker bioassay utilizing HE4 and CA125 for the prediction of ovarian cancer in patients with a pelvic mass. Gynecol Oncol 2009; 112: 40-46.

8. Badgwell D, Bast RC Jr. Early detection of ovarian cancer. Dis Markers 2007; 23: 397-410.

9. Moore RG, Miller MC, Steinhoff MM, et al. Serum HE4 levels are less frequently elevated than CA125 in women with benign gynecologic disorders. Am J Obstet Gynecol 2012; 206: 351.e1-8.

10. DiSaia PJ, Creasman WT. Epithelial ovarian cancer. In: Clinical Gynecologic Oncology. DiSaia PJ, Creasman WT (eds.). $5^{\text {th }}$ ed. Mosby-Year Book, St Louis 1997; 282-350.

11. Piver MS, Hempling RE. Ovarian cancer: etiology, screening, prophylactic oopherectomy, and surgery. In: Te Linde's Operative Gynecology. Rock JA, Thompson JD (eds.). $8^{\text {th }}$ ed. Lippincott-Raven Publishers, Philadelphia, New York 1997; 1557-1568.

12. Rustin GJS, van der Burg MEL, Berek JS. Tumour markers. Ann Oncol 1993; 4: 71-77.

13. Geomini P, Kruitwagen R, Bremer GL, et al. The accuracy of risk scores in predicting ovarian malignancy: a systematic review. Obstet Gynecol 2009; 113 (2 Pt 1): 384-394

14. Jacobs I, Oram D, Fairbanks J, et al. A risk of malignancy index incorporating CA 125, ultrasound and menopausal status for the accurate preoperative diagnosis of ovarian cancer. Br J Obstet Gynaecol 1990; 97: 922-929.

15. Tingulstad S, Hagen B, Skjeldestad FE, et al. Evaluation of a risk of malignancy index based on serum CA125, ultrasound findings and menopausal status in the pre-operative diagnosis of pelvic masses. $\mathrm{Br} J \mathrm{Ob}$ stet Gynaecol 1996; 103: 826-831.

16. Tingulstad S, Hagen B, Skjeldestad FE, et al. The risk-of-malignancy index to evaluate potential ovarian cancers in local hospitals. Obstet Gynecol 1999; 93: 448-452. 
17. Torres JC, Derchain SF, Faundes A, et al. Risk-of-malignancy index in preoperative evaluation of clinically restricted ovarian cancer. Sao Paulo Med J 2002; 120: 72-76.

18. Clauss A, Lilja H, Lundwall A. A locus on human chromosome 20 contains several genes expressing protease inhibitor domains with homology to whey acidic protein. Biochem J 2002; 368 (Pt 1): 233-242.

19. Clauss A, Lilja H, Lundwall A. The evolution of a genetic locus encoding small serine proteinase inhibitors. Biochem Biophys Res Commun 2005 333: 383-389.

20. Lu R, Sun X, Xiao R, et al. Human epididymis protein 4 (HE4) plays a key role in ovarian cancer cell adhesion and motility. Biochem Biophys Res Commun 2012; 419: 274-280.

21. Drapkin R, von Horsten $\mathrm{HH}$, Lin $\mathrm{Y}$, et al. Human Epididymis Protein 4 (HE4) is a secreted glycoprotein that is overexpressed by serous and endometrioid ovarian carcinomas. Cancer Res 2005; 65: 2162-2169.

22. Galgano MT, Hampton GM, Frierson HF Jr. Comprehensive analysis of HE4 expression in normal and malignant human tissues. Mod Patho 2006; 19: 847-853.

23. Moore RG, Miller MC, Eklund EE, et al. Serum levels of the ovarian cancer biomarker HE4 are decreased in pregnancy and increase with age. Am J Obstet Gynecol 2012; 206: 349.e1-7.

24. Hallamaa M, Suvitie P, Huhtinen K, et al. Serum HE4 concentration is not dependent on menstrual cycle or hormonal treatment among endometriosis patients and healthy premenopausal women. Gynecol Oncol 2012; 125: 667-672.

25. Chang $X$, Ye X, Dong L, et al. Human epididymis protein 4 (HE4) as a serum tumor biomarker in patients with ovarian carcinoma. Int J Gynecol Cancer 2011; 21: 852-858.

26. Moore RG, Jabre-Raughley M, Brown AK, et al. Comparison of a nove multiple marker assay vs the Risk of Malignancy Index for the prediction of epithelial ovarian cancer in patients with a pelvic mass. Am J Obstet Gynecol 2010; 203: 1-6.

27. Jacob F, Meier M, Caduff R, et al. No benefit from combining HE4 and CA125 as ovarian tumor markers in a clinical setting. Gynecol Oncol 2011; 121: 487-491.

28. Van Gorp T, Cadron I, Despierre E, et al. HE4 and CA125 as a diagnostic test in ovarian cancer: prospective validation of the Risk of Ovarian Malignancy Algorithm. Br J Cancer 2011; 104: 863-870.

29. Yang Z, Wei C, Luo Z, et al. Clinical value of serum human epididymis protein 4 assay in the diagnosis of ovarian cancer: a meta-analysis. Onco Targets Ther 2013; 6: 957-966.

30. Li F, Tie R, Chang K, et al. Does risk for ovarian malignancy algorithm excel human epididymis protein 4 and CA125 in predicting epithelia ovarian cancer: a meta-analysis. BMC Cancer 2012; 12: 258

31. Lin J, Qin J, Sangvatanakul V. Human epididymis protein 4 for differential diagnosis between benign gynecologic disease and ovarian cancer: a systematic review and meta-analysis. Eur J Obstet Gynecol Reprod Biol 2013; 167: 81-85.

32. http://www.fda.gov/NewsEvents/Newsroom/PressAnnouncements/ 2009/ucm182057.htm

33. http:// www.ova-1.com/

34. http://www.prnewswire.com/news-releases/vermillion-announces positive-top-line-results-from-the-ova500-multi-center-study-led-byuc-irvine-164249626.html

35. Bast RC Jr, Skates S, Lokshin A, et al. Differential diagnosis of a pelvic mass: improved algorithms and novel biomarkers. Int J Gynecol Cancer 2012; 22 Suppl 1: S5-8.
36. Ueland FR, Desimone CP, Seamon LG, et al. Effectiveness of a multivariate index assay in the preoperative assessment of ovarian tumors. Obstet Gynecol 2011; 117: 1289-1297.

37. Ware Miller R, Smith A, DeSimone CP, et al. Performance of the American College of Obstetricians and Gynecologists' ovarian tumor referral guidelines with a multivariate index assay. Obstet Gynecol 2011; 117: 1298-1306

38. Nam EJ, Yoon H, Kim SW, et al. MicroRNA expression profiles in serous ovarian carcinoma. Clin Cancer Res 2008; 14: 2690-2695.

39. Dahiya N, Morin PJ. MicroRNAs in ovarian carcinomas. Endocr Relat Cancer 2010; 17: F77-F89.

40. Köbel M, Kalloger SE, Boyd N, et al. Ovarian carcinoma subtypes are different diseases: implications for biomarker studies. PLoS Med 2008; 5: e232.

41. Mitchell PS, Parkin RK, Kroh EM, et al. Circulating microRNAs as stable blood-based markers for cancer detection. Proc Natl Acad Sci USA 2008; 105: 10513-10518.

42. Häusler SF, Keller A, Chandran PA, et al. Whole blood-derived microRNAs profiles as potential new tools for ovarian cancer screening. Br J Cancer 2010; 103: 693-700.

43. Chen X, Ba Y, Ma L, et al. Characterization of microRNAs in serum: a novel class of biomarkers for diagnosis of cancer and other diseases. Cell Res 2008; 18: 997-1006.

44. Kroh EM, Parkin RK, Mitchell PS, Tewari M. Analysis of circulating microRNA biomarkers in plasma and serum using quantitative reverse transcription-PCR (qRT-PCR). Methods 2010; 50: 298-301.

45. Zheng H, Liu JY, Song FJ, Chen KX. Advances in circulating microRNAs as diagnostic and prognostic markers for ovarian cancer. Cancer Biol Med 2013; 10: 123-130.

46. Suryawanshi S, Vlad AM, Lin HM, et al. Plasma microRNAs as novel biomarkers for endometriosis and endometriosis-associated ovarian cancer. Clin Cancer Res 2013; 19: 1213-1224.

47. Lawrie CH, Gal S, Dunlop HM, et al. Detection of elevated levels of tumour-associated microRNAs in serum of patients with diffuse large B-cell lymphoma. Br J Haematol 2008; 141: 672-675.

48. Resnick KE, Alder H, Hagan JP, et al. The detection of differentially expressed microRNAs from the serum of ovarian cancer patients using a novel real-time PCR platform. Gynecol Oncol 2009; 112: 55-59.

49. Hu Z, Chen X, Zhao Y, et al. Serum microRNA signatures identified in a genome-wide serum microRNA expression profiling predict survival of non-small-cell lung cancer. J Clin Oncol 2010; 28: 1721-1726.

50. Taylor DD, Gercel-Taylor C. MicroRNA signatures of tumor-derived exosomes as diagnostic biomarkers of ovarian cancer. Gynecol Oncol 2008; 110: 13-21.

51. Kan CW, Hahn MA, Gard GB, et al. Elevated levels of circulating microRNA-200 family members correlate with serous epithelial ovarian cancer. BMC Cancer 2012; 12: 627-636.

52. Chung YW, Bae HS, Song JY, et al. Detection of microRNA as novel biomarkers of epithelial ovarian cancer from the serum of ovarian cancer patient. Int J Gynecol Cancer 2013; 23: 673-679.

53. Ji T, Zheng ZG, Wang FM, et al. Differential microRNA expression by Solexa sequencing in the sera of ovarian cancer patients. Asian Pac J Cancer Prev 2014; 15: 1739-1743.

54. Shapira I, Oswald M, Lovecchio J, et al. Circulating biomarkers for detection of ovarian cancer and predicting cancer outcomes. BJC 2014; 110: 976-983. 